\title{
Foreword: Coping with sex chromosome imbalance
}

\author{
Edith Heard • Laura Carrel
}

Published online: 11 September 2009

(C) Springer Science + Business Media B.V. 2009

This special issue is devoted to the topic of dosage compensation. The dramatic phenotypes that are associated with most autosomal monosomies or trisomies illustrate the importance of maintaining appropriate gene dosage in diploid organisms. Buffering and compensating for such genetic imbalance clearly represents a challenge for complex eukaryotes. In sexually dimorphic species with chromosomebased mechanisms of sex determination $(\mathrm{XX} / \mathrm{XY}$ and $\mathrm{ZW} / \mathrm{ZZ}$ ), the contrasting doses of sex chromosomes in males and females would be expected to introduce a large-scale imbalance in levels of gene expression. This imbalance would be at two levels. First, the dosage differences between the sexes (XX versus XY or ZW versus $\mathrm{ZZ}$ ); second, the dosage difference between sex chromosomes and autosomes in the heterogametic sex (XY males, or ZW females) where only a single copy of the $\mathrm{X}$ or $\mathrm{Z}$ chromosome is present, in contrast to two copies of all autosomes.

Responsible Editor: Edith Heard.

E. Heard $(\bowtie)$

Mammalian Developmental Epigenetics Group, Institut Curie, CNRS UMR3215 INSERM U934,

26 rue d'Ulm,

75248 Paris Cedex 05, France

e-mail: Edith.Heard@curie.fr

L. Carrel

Department of Biochemistry and Molecular Biology,

Penn State College of Medicine,

Hershey, PA 17033, USA
However, sex chromosome imbalances are usually well tolerated-how can this be?

It is now clear that in many organisms the evolution of sex chromosomes from an ancestral autosome pair must have been accompanied by the evolution of dosage compensation strategies, in order to equalize sex-linked gene expression in males and females. The existence of such strategies was first noted many decades ago. In 1932, the issue was first raised for D. melanogaster by Muller. In 1967, Ohno predicted that "...during the course of evolution, an ancestor to placental mammals must have escaped a peril resulting from the hemizygous existence of all the $\mathrm{X}$-linked genes in the male by doubling the rate of product output of each X-linked gene". It is now clear that up-regulation of the male $\mathrm{X}$ chromosome to achieve equivalent levels to autosomes is an evolutionarily conserved strategy found in worms, flies and mammals. In 1961 Mary Lyon published her seminal paper, outlining the process of X-chromosome inactivation in mammals, that enables equivalence of X-linked gene dosage between the sexes to be obtained through the silencing and heterochromatinization of one of the two $\mathrm{X}$ chromosomes in females during early development. Numerous alternative strategies of dosage compensation strategies were subsequently discovered in other organisms with $\mathrm{XY} / \mathrm{XX}$ dimorphic sex chromosomes. In flies up-regulation of the $X$ chromosome occurs only in males, thus dealing with both the sex-specific and $\mathrm{X}$-autosomal dosage problems in one fell swoop. In 
worms, on the other hand, both $\mathrm{X}$ chromosomes are down-regulated by about half in XX hermaphrodites. Dosage compensation in organisms with a ZZ/ZW sex chromosome constitution such as birds and lepidoptera is more enigmatic, but there is evidence that dosage compensation strategies must exist, at least on a gene-by-gene basis. Indeed, the diversity of mechanisms in the organisms so far analysed is startling and there is an increasing realization that even within taxa, alternative dosage compensation mechanisms can evolve rapidly. It is only in recent years, with the availability of whole genome sequences, as well as techniques enabling identification and detection of specific protein and RNA molecules, that the mechanistic basis of this plethora of dosage compensation strategies is starting to be unraveled.

In this special issue of Chromosome Research, we provide a unique collection of reviews that covers dosage compensation at several different levels. The mechanisms underlying some of the best-studied dosage compensation strategies in mammals, worms and flies, are discussed and the evolutionary implications of dosage compensation are explored. Several questions are addressed in these reviews. First, why do dosage compensation strategies evolve in the first place? Their evolution is clearly linked to the forces driving the evolution of sex chromosomes. However, the forces driving dosage compensation are also unique and the requirements for dosage compensation seem to vary dramatically, even between closely related species. Why is this? Did dosage compensation evolve specifically for a subset of sex chromosomal genes? And, is dosage compensation of such genes actually important at all times during development and/or in somatic tissues? Another important series of questions concerns how dosage compensation strategies evolved. Are there common mechanisms of dosage compensation-i.e. has there been repeated use of similar strategies during evolution? Or does each system require invention of a new strategy? Diversity certainly seems to be the rule, as the set of sequences and molecules exploited for transcriptional up- or down regulation, and for targeting and spreading chromatin changes, seem to be very different between systems. The fine-tuning of gene expression that dosage compensation entails may be either at the transcriptional or post-transcriptional levels. For those systems where control is clearly at the transcriptional level (flies and mammals), although proteins or RNA molecules may have evolved to ensure the sex-chromosome specific targeting of chromatin-associated factors, the latter come largely from the pre-existing repertoire of chromatin modifying and remodeling complexes. A further set of questions concerns the impact of dosage compensation on subsequent sex chromosome evolution and the constraints that this can impose. Finally, dosage compensation extends beyond sex chromosomes. It is important to consider the impact of partially or fully duplicating a genome on the overall balance of gene dosage. The recent availability of sequenced genomes is revealing that most, if not all, result from one or several ancestral whole genome duplication events and contain multiple traces of recent, as well as old, segmental duplications. Although duplications are essential for the evolution of novel, specialized gene functions as postulated by Ohno, whole genome or segmental duplications also generate potential gene dosage imbalances. We can only speculate at this stage as to the potential compensation or buffering mechanisms that must come into play when such large-scale events take place.

The series begins with a review article by Beatriz Vicoso and Doris Bachtrog on the evolutionary considerations of dosage compensation and sex chromosomes. Heteromorphic sex chromosomes are believed to evolve from ancestral autosome pairs. In the case of XY, acquisition of a male-determining gene on an autosome is thought to lead to formation of a proto-Y chromosome. Subsequently, malebeneficial mutations accumulate on this chromosome. Suppression of recombination on the $\mathrm{Y}$ will be selected to protect linkage between the maledetermining gene and male-beneficial genes, but this results in the gradual degeneration of the $\mathrm{Y}$, with an accumulation of deleterious mutations and transposable elements in the absence of purifying selection on the non-recombining chromosome. The degeneration of the $\mathrm{Y}$ leads to males with only one functional copy of $\mathrm{X}$ or $\mathrm{Y}$ genes and the resulting imbalance of $\mathrm{X}$-linked versus autosomal gene expression would favor the evolution of mechanisms that increase expression of genes on the single $\mathrm{X}$ in males. The authors propose that this is most likely the first step in the evolution of dosage compensation and that only subsequently would the evolution of X-linked repression in females be necessary, in situations (such as in 
worms and mammals) where X-chromosome upregulation is not restricted to males. A similar scenario may apply to the evolution of ZW pairs, although less is known about their evolution and also their dosage compensation strategies. Indeed, in birds it is unclear to what extent there is dosage compensation at least on a chromosome-wide level. In this context, the authors discuss several important differences that may exist in the evolution of dosage compensation when the female, as opposed to the male, is the heterogametic sex. The authors also compare the origins of dosage compensation genes between different taxa and discuss the remarkable diversity and rapid evolution of such genes.

The six articles that follow cover the dosage compensation strategies found in different animals. First, Erinc Hallancli and Asifa Akhtar review the 2-fold up-regulation of the male $\mathrm{X}$ chromosome in Drosophila melanogaster. The Sxl protein, which is only expressed in XX females, prevents formation of the dosage compensation complex (DCC), thus restricting dosage compensation to males. The DCC includes several male specific lethal (MSL) proteins as well as two non-coding RNAs, Rox1 and Rox2. The DCC decorates the male $\mathrm{X}$ chromosome by binding to hundreds of sites along the $\mathrm{X}$ chromosome resulting in altered chromatin structure, notably histone H4 Lys16 acetylation. Recent genome-wide analyses have shed new light into this process. The DCC is thought to initially target a subset of high affinity "entry sites" on the X chromosome, followed by local spreading in cis. The authors also discuss new data showing that MSL proteins mediate the finely tuned, 2-fold up-regulation of the X-chromosome not simply via histone acetyltransferase (HAT) activity, but also through nuclear compartmentalization by enabling proximity with the nuclear pore, cross-talk with chromatin remodelers, heterochromatin proteins, and RNA degradation pathways. Nevertheless, despite the essential role of MSL proteins in this process, recent experiments reveal that even closely related Drosophila species, display highly asymmetric rapid evolution of MSL genes. Next, Gyorgyi Csankovszki, Emily Petty and Karishma Collette provide an in-depth review of our current understanding of dosage compensation in the nematode, C. elegans, which involves an approximately two-fold down regulation of both $\mathrm{X}$ chromosomes in XX cells. This is achieved by binding of a condensin-like DCC to both $\mathrm{X}$ chromosomes in hermaphrodites to down-regulate gene expression. Thanks to careful genetic studies and genome wide mapping of DCC sites of enrichment, the specific recruiting elements (rex sites) that target the DCC to the $\mathrm{X}$ chromosome are now known. Once bound the DCC then spreads or is dispersed to secondary (dox) sites. However, a recent surprise came from the finding that unlike in Drosophila, where DCC target sites correspond to the genes being regulated, in C. elegans, it turns out that down-regulated gene promoters are not necessarily the primary targets of the DCC! Thus, the complex may act in a chromosome-wide manner, rather than on a gene-by-gene basis. The fact that the DCC is formed in part by condensin proteins, strongly suggests that it may act via a chromosomal condensation mechanism, analogous to mitotic chromosome condensation, although the exact mechanism of action of the DCC remains tantalizingly unclear. Indeed, evidence suggests that RNA processing rather than changes in chromatin structure may be important in this system, where a 2-fold down regulation, as opposed to full shut-down, is required.

The next few articles deal with dosage compensation in mammals. In most eutherian mammals, one of the two $\mathrm{X}$ chromosomes becomes inactivated at random during early development. In marsupials, $\mathrm{X}$ inactivation also occurs, but affects only the paternal $\mathrm{X}$ chromosome. In monotremes, the situation is less clear and appears to involve stochastic gene silencing of some regions. Three reviews discuss the key master locus in eutherian mammals, the $\mathrm{X}$-inactivation center (XIC). This locus initiates the onset of X inactivation by producing the non-coding Xist RNA which coats and mysteriously silences chromatin in cis. Surprisingly however, this RNA is not well conserved and other mammals appear to exploit different strategies to achieve the same end result. Katie Prothero, Jill Stahl and Laura Carrel discuss the recently discovered evidence for X-chromosome up-regulation in male and female mammals, in order to equalize dosage relative to autosomes. They also discuss X-chromosome inactivation in female placental mammals, particularly in humans, where many genes are in fact not X inactivated, and thus are not necessarily fully dosage compensated. These genes that "escape" X inactivation have interesting implications not only in terms of the impact they can have on normal traits and disorders that differ between men and women, but also from a mechanistic point of view, they provide insight into how $\mathrm{X}$ inactivation 
spreads and is maintained on the inactive $\mathrm{X}$. Inadequate gene dosage of some of these "escapee" genes explains in part the phenotypic consequences of $\mathrm{X}$ chromosome monosomy, a topic that is dealt with in more detail in the review by Carolyn Bondy and Clara Cheng. They describe the phenotypic effects of $\mathrm{X}$ monosomy in humans and other mammals, in particular on normal development, fertility and longevity, and discuss the possible causes of these effects in terms of $\mathrm{X}$ chromosome gene dosage.

The review by Ikuhiro Okamoto and Edith Heard discusses the developmental dynamics of $\mathrm{X}$ inactivation and the mechanistic and evolutionary divergence in $\mathrm{X}$ inactivation between mammals in a developmental context. In particular the evolutionary origins of imprinted $\mathrm{X}$ inactivation which is found in marsupials, but also in mice are discussed. In mice, $\mathrm{X}$ inactivation is only subject to imprinting during early development and is dependent on imprinted Xist expression, but not on meiotic sex chromosome inactivation (MSCI), which occurs during meiosis in the male germ line. The situation may be rather different in marsupials, which have no Xist gene. Indeed, the evolution of imprinted $\mathrm{X}$ inactivation in mice and marsupials are likely to have been driven independently by different forces. Further studies on early development of different mammals should reveal the degree to which dosage compensation is subject to parent-specific modulation and will undoubtedly provide more surprises in terms of evolutionary diversity linked to early developmental requirements. Janine Deakin, Julie Chaumeil, Timothy Hore and Jennifer Graves review our current knowledge on the fascinating dosage compensation strategies in marsupials and monotremes. As mentioned above, marsupial $\mathrm{X}$ inactivation does not rely on the Xist gene. Whether another Xist-like locus is involved in triggering this process in these mammals remains to be seen. It is unclear to what extent $\mathrm{X}$ inactivation in marsupials is a chromosome-wide as opposed to a gene-by-gene silencing phenomenon. Furthermore, little is known about the trigger for this process and the epigenetic mechanisms that maintain it. In fact, some marsupials push $\mathrm{X}$ inactivation to an extreme and actually eliminate the paternal inactive $\mathrm{X}$ in females (and the $\mathrm{Y}$ in males). Monotremes are a very curious mixture of reptilian and mammalian features. Remarkably, their sex chromosomes (five $\mathrm{X}$ and five $\mathrm{Y}$ chromosomes) show no homology with therian sex chromosomes but more closely resemble bird ZW chromosomes! Thus it will not be a surprise if their dosage compensation strategy is different to those of marsupials and eutherian mammals. So far evidence points to some, but not all genes, displaying monoallelic gene expression. However, whether this is clonal and whether it is stochastic or parent-of-origin specific remain to be found. Stochastic, monoallelic gene expression may also be a feature of bird dosage compensation mechanisms, as discussed in the review by Heather McQueen and Michael Clinton. In this article they re-evaluate data for $\mathrm{Z}$ chromosome activity in birds and propose that contrary to previous views, some genes do show dosage compensation, albeit it at varying levels. In particular, a cluster of genes on the $\mathrm{Z}$ chromosome shows robust dosage compensation and this may involve non-coding RNAs. Whatever the mechanism of dosage compensation, the evidence suggests that unlike in mammals, this does not involve monoallelic expression in the homogametic sex (males), but instead possibly an up-regulation of the single $\mathrm{Z}$ chromosome in females, similar to the situation for the male $\mathrm{X}$ in flies. Once more, genome-wide and molecular studies promise exciting new insights into dosage compensation of birds in the future.

Extending beyond sex chromosomes, Patrick Edger and Chris Pires explore the impact of dosage sensitivity on the fate of nuclear genes. Whole genome duplications (WGDs) followed by gene loss (i.e. diploidization) have occurred recurrently in the evolution of higher eukaryotes. Gene retention is biased to specific functional gene categories during diploidization. By analysing extant genomes and their composition the authors present evidence that only a subset of genes, such as transcription factors, are sensitive to dosage, and these are often retained after whole genome duplication. On the other hand such genes are eliminated following segmental duplication. In this review, different theories that address the fate of nuclear genes following duplication events are presented and the various mechanisms of dosagecompensation that have evolved to alleviate harmful dosage-imbalances are reviewed. The authors focus on an example in flowering plants to speculate on the possibility that preferential retention of dosage sensitive genes and loss of dosage insensitive genes play a role in facilitating speciation and the development of morphological complexity in eukaryotes. 
While the concept of dosage compensation is longstanding, recent access to genomic sequence and the use of new technologies is now providing us with a glimpse of the amazing diversity of strategies and molecular mechanisms involved in different organisms. Finding out how gene dosage is dealt with is an issue that touches on many different aspects of evolutionary, cellular and developmental biology, and extends well beyond sex chromosomes. We hope that after reading the articles in this issue you will share some of our fascination for dosage compensation strategies. We would like to thank all authors for their excellent contributions to this special issue and all referees for their valuable suggestions on the manuscripts. Our special thanks go to Herbert Macgregor, Editor-in-Chief of Chromosome Research, and his editorial board for the enthusiasm and support for this project. 